\title{
Speed-Density Model of Interrupted Traffic Flow Based on Coil Data
}

\author{
Chen Yu, ${ }^{1}$ Jiajie Zhang, ${ }^{1}$ Dezhong Yao, ${ }^{1}$ Ruiguo Zhang, ${ }^{2}$ and Hai Jin ${ }^{1}$ \\ ${ }^{1}$ Services Computing Technology and System Lab, Big Data Technology and System Lab, Cluster and Grid Computing Lab, \\ School of Computer Science and Technology, Huazhong University of Science and Technology, Wuhan 430074, China \\ ${ }^{2}$ Siemens Ltd., China Corporate Technology, Wireless Technology and Web of System Wuhan Innovation Center, Wuhan 430074, China \\ Correspondence should be addressed to Chen Yu; yuchen@hust.edu.cn
}

Received 2 September 2016; Accepted 13 November 2016

Academic Editor: Beniamino Di Martino

Copyright (C) 2016 Chen Yu et al. This is an open access article distributed under the Creative Commons Attribution License, which permits unrestricted use, distribution, and reproduction in any medium, provided the original work is properly cited.

\begin{abstract}
As a fundamental traffic diagram, the speed-density relationship can provide a solid foundation for traffic flow analysis and efficient traffic management. Because of the change in modern travel modes, the dramatic increase in the number of vehicles and traffic density, and the impact of traffic signals and other factors, vehicles change velocity frequently, which means that a speed-density model based on uninterrupted traffic flow is not suitable for interrupted traffic flow. Based on the coil data of urban roads in Wuhan, China, a new method which can accurately describe the speed-density relation of interrupted traffic flow is proposed for speed fluctuation characteristics. The model of upper and lower bounds of critical values obtained by fitting the data of the coils on urban roads can accurately and intuitively describe the state of urban road traffic, and the physical meaning of each parameter plays an important role in the prediction and analysis of such traffic.
\end{abstract}

\section{Introduction}

Flow, speed, and density are known as the basic elements of traffic flow theory. Flow can measure the number of vehicles and the demand for traffic infrastructure. Speed is an important control index in road planning, and it is also an evaluation index of vehicle operation efficiency. Density reflects the intensity of the vehicles on the road and determines traffic management and control measures. The relationships between flow, speed, and density called fundamental diagrams play a very important role in traffic flow theory and traffic engineering. For example, the speed-flow relationship can be used in highway capacity analysis in order to determine the highway service quality, and the speed-density relationship can reflect dynamic change in traffic flow, which can be used to study the disturbance propagation between vehicles. Therefore, sound mathematical models provide a solid foundation for traffic flow analysis and efficient traffic management. The relationship between speed and density which can reflect the quality of service received from the road is attracting considerable research attention.
The earliest speed-density model was a linear model proposed by Greenshields et al. [1] in 1935. The linear model overlaps and classifies the observed data groups, which is proved to be unreasonable, and observation time is a holiday, with a narrow range of representations, so there are some deviations between the derived speed-density relation and the actual situation. Later, the relationship between speed and density was studied in greater depth, and the Greenberg logarithmic model, Edie model, Underwood exponent model, PipesMunjal model, modified Greenshields model, Newell model, and so forth, emerged in turn [2,3]. Heydecker and Addison [4] studied the relationship between speed and density under various speed limits and found that zero speed induces traffic jams, not the other way around. Ma et al. [5] derived a general logistic model of traffic flow characteristics, which includes several traffic flow parameters with clear physical meanings and analyzed the effects of the parameters on speed-density logistic curves. The experimental results showed that this model can well describe the traffic flow characteristics in different states. Shao et al. [6] proposed a speed-density model 
under congested traffic conditions combined with the minimum safety spacing constraint, and the experimental results showed that the absolute error of this model was smaller than that of other models fitting the traffic data of two freeways. Wang et al. [7] proposed a family of speed-density models with different numbers of parameters with important physical significance and got good performance in the final experiment.

All of the above studies are based on continuous traffic flow data. These data, also called uninterrupted traffic flow, are traffic flow with no effect of external fixation factors, such as freeway, urban expressway, and so forth. Discontinuous traffic flow, referred to as interrupted traffic flow, is periodically influenced by external fixation factors. The most common interrupted traffic flow is originated by signal lamps of urban intersections. Because of the variety of vehicle types, the periodic effect of signal lamps, shunts in the canal section, and other factors, the characteristics of interrupted traffic flow are very complex compared with uninterrupted traffic flow. In addition, the city is still in a rapid increase in population and, with the development of economy, people are more inclined to self-driving travel, thus more and more vehicles and more and more congestion in the city, which leads to the increase of travel time, the growth of fuel consumption [8], the aggravation of environmental pollution, and other awful issues $[9,10]$. Compared with the highway, the urban road has a strong influence on the individual, society, and the environment. Therefore, further study of the characteristics of interrupted traffic flow to provide support for management decisions is particularly important.

Research on interrupted traffic flow has attracted a lot of attention [11-15]. Many scholars see traffic flow located at a certain distance from the intersection as continuous traffic flow, believing that it can be described by continuous traffic flow models. Some of the literature $[16,17]$ suggests, however, that because of the short distance between intersections in the city and the influence of signal lamps, there are differences between traffic flow located at a certain distance from the intersection and the traffic flow of freeways. Because traffic data are difficult to obtain and for other objective reasons, only a few scholars focus on the speed-density model of discontinuous traffic flow. Wang et al. [18] introduced a fourparameter logit model for complete data fitting and established a speed-density logit model for left-turning, straight, and right-turning traffic flow. However, the experimental data were obtained by VISSIM simulation, and the simulation parameters were not accurate enough to depict the complex city road environment, so the experimental results have certain limitations. Wang et al. [19] thought that the stochastic model would contain more traffic information and put forward the stochastic speed-density model. This stochastic model can generate a probabilistic traffic flow model and can achieve real-time traffic prediction.

In order to provide favorable data analysis and presentation for city traffic, thus to provide decision support for intelligent transportation, characterizing the speed-density relationship of interrupted traffic flow more accurately is full of importance. By analyzing a large amount of data, we propose a description method for a speed-density relationship model which is suitable for discontinuous traffic flow, using the upper and lower curves to describe the upper and lower bounds of velocity values. Because of the discrepant characteristics of the traffic flow in the outer and inner lanes, the coil data of the outer and inner lanes are analyzed and verified.

\section{Speed-Density Model}

Three basic parameters (flow $q$, speed $u$, and density $k$ ) are the core content of the traffic flow model. The three have the following relationship:

$$
q=k \times u
$$

that is, flow is the product of density and speed. The relationship between two parameters of the three is of great significance in traffic flow, and the relationship between speed and density has received a lot of research attention. Greenshields et al. was an early researcher, who proposed the speed-density linear relationship [1]:

$$
u=u_{f} \times\left(1-\frac{k}{k_{j}}\right),
$$

where $u_{f}$ is the speed of free flow, that is, the speed of vehicles unimpeded when the traffic density tends to zero, and $k_{j}$ is the density of block flow, that is, the density when the traffic flow is blocked and cannot move. As shown in Figure 1, when $k=0$, the speed can reach the theoretical maximum value, namely, the free flow velocity $u_{f}$. The area surrounded by the abscissa, the ordinate of any point on the line, and the coordinate origin is the traffic flow.

Equation (2) can change to

$$
k=k_{j} \times\left(1-\frac{u}{u_{f}}\right) .
$$

Respectively, introduce (2) and (3) into (1), and we get

$$
\begin{aligned}
& q=u_{f} \times\left(k-\frac{k^{2}}{k_{j}}\right), \\
& q=k_{j} \times\left(u-\frac{u^{2}}{u_{f}}\right) .
\end{aligned}
$$

Equations (4) illustrate that $q-k$ and $q-u$ are quadratic function relations, as shown in Figure 1.

The linear model is too simple, and there are many deficiencies. In order to improve the model, scholars have proposed models based on the linear model but with a higher degree of accuracy. Table 1 lists results for the speed-density model, including the Greenberg model, Underwood model, Northwestern model, Newell's model, Pipes-Munjal model, Drew model, Modified Greenshields model, Del Castillo and Benitez model, Van Aerde model, MacNicholas model. These models with the parameters of important physical meaning provide good results.

Wang et al. [19] established a speed-density logit probability model with four parameters. Wang et al. used VISSIM 
TABLE 1: Speed-density models.

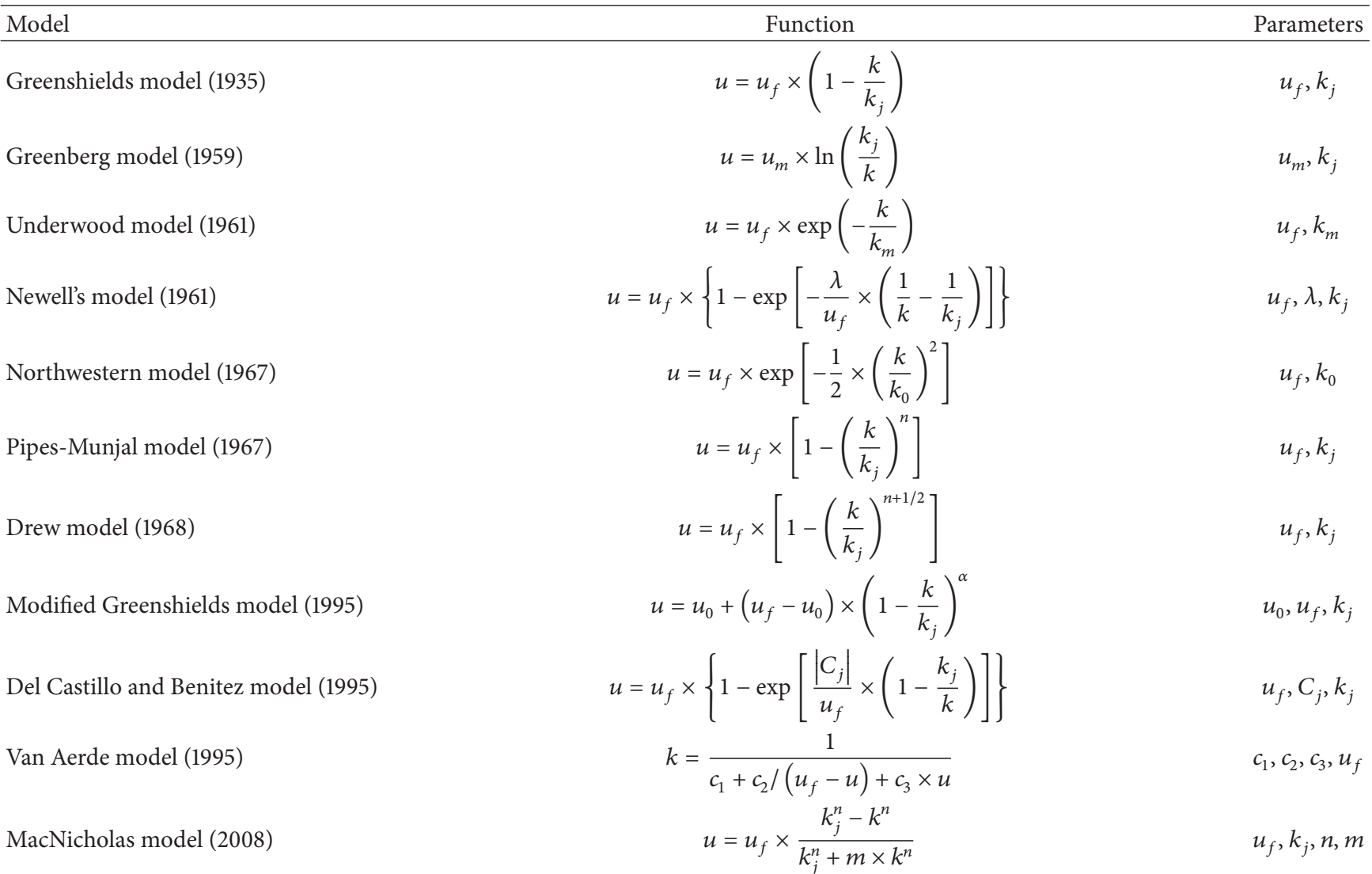

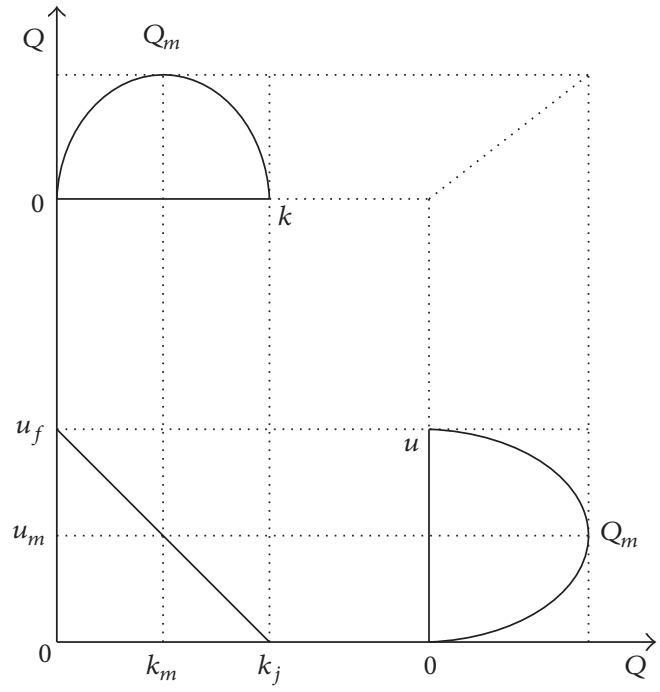

Figure 1: The mapping of speed-density, flow-density, and speedflow.

simulation software to set up and change six parameters of road traffic, including section length $L$, stretch section length $l$, cart rate $\alpha$, signal period $C$, the ratio of the time span of left-turn green signal to signal period $\lambda_{l}$, and the ratio of the time span of the straight green signal to signal period $\lambda_{s}$, and established 22 groups of parameters. The simulation results showed that the relationship between speed and density presents an inverse S curve. Therefore, a four-parameter logit model is proposed here to describe the speed-density inverse $S$ curve, and its expression is as follows:

$$
u=u_{\min } \times \frac{u_{\max }-u_{\min }}{1+\exp \left(\left(N-N_{w}\right) / \theta\right)},
$$

where $u_{\text {min }}$ is the mean value of the minimum speed, $u_{\max }$ is the mean value of the maximum speed, $N$ is the flow value of a section, $N_{w}$ is the flow value at the inflection point of the curve, and $\theta$ is a parameter determining curve shape.

Then, the data obtained from the 22 groups of simulation parameters were fitted. The four parameters $\left(u_{\min }, u_{\max }, N_{w}\right.$, and $\theta$ ) were calculated for each simulation environment. $N_{w}$ and $\theta$ were, respectively, fitted in left-turn, straight, and rightturn cases, and the fitting results are as follows:

$$
\begin{aligned}
& N_{w} \\
& = \begin{cases}0.7146, & \text { left-turning } \\
-0.2231 \alpha+0.1989, & \text { straight } \\
-0.00011 L+0.0078 l-0.2113 \alpha+0.2282, & \text { right-turning, }\end{cases} \\
& \theta= \begin{cases}0.0664, & \text { left-turning } \\
-0.000032 L+0.085 l, & \text { straight } \\
0.045 l, & \text { right-turning. }\end{cases}
\end{aligned}
$$




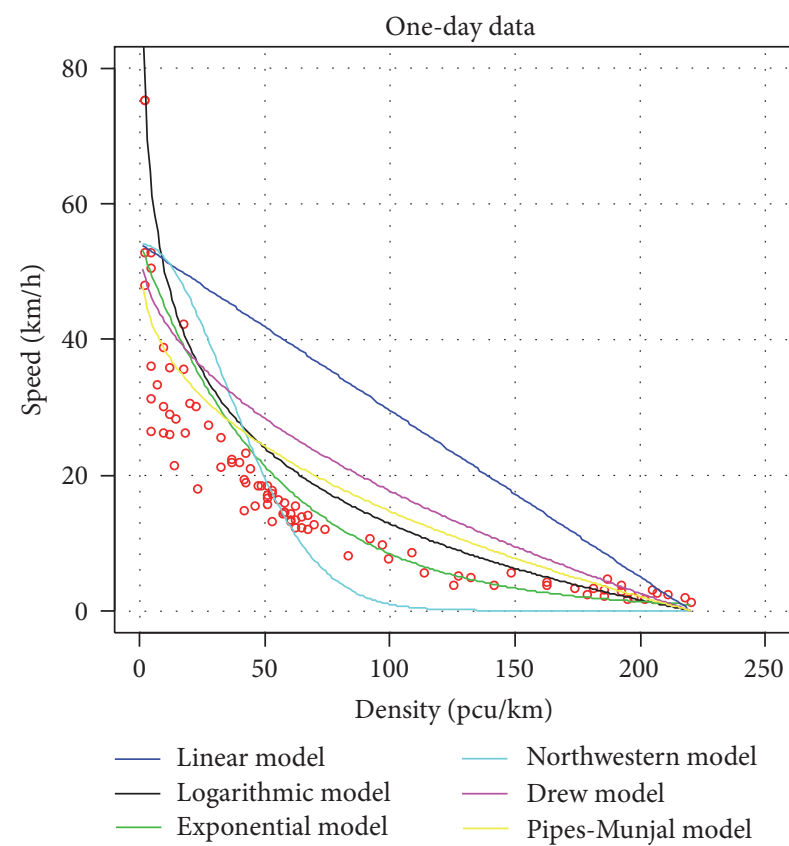

(a) Graph of one-day data

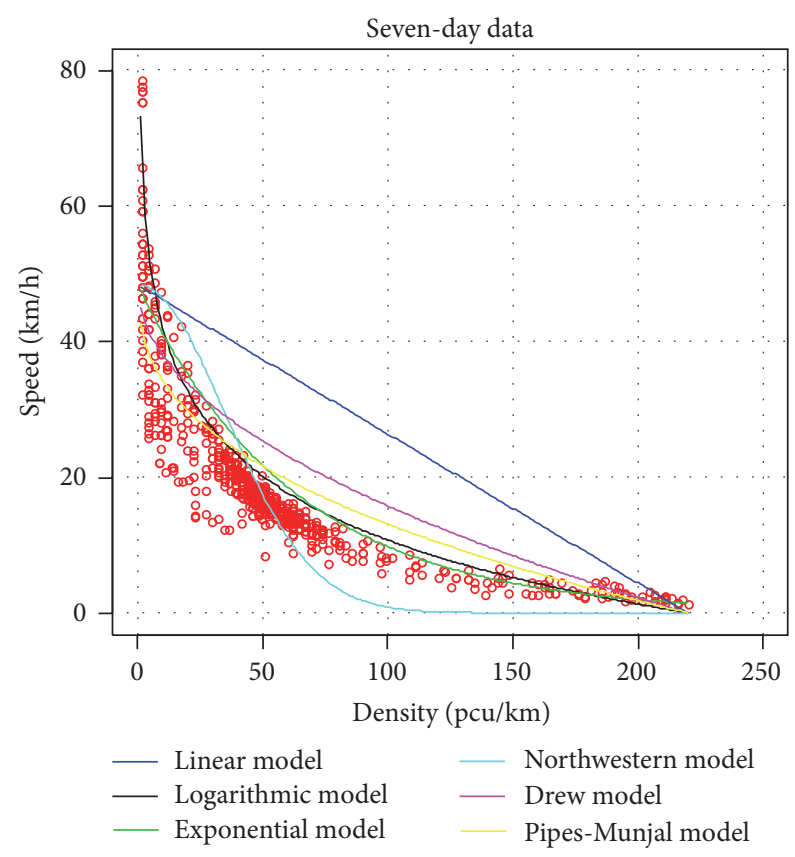

(c) Graph of seven-day data

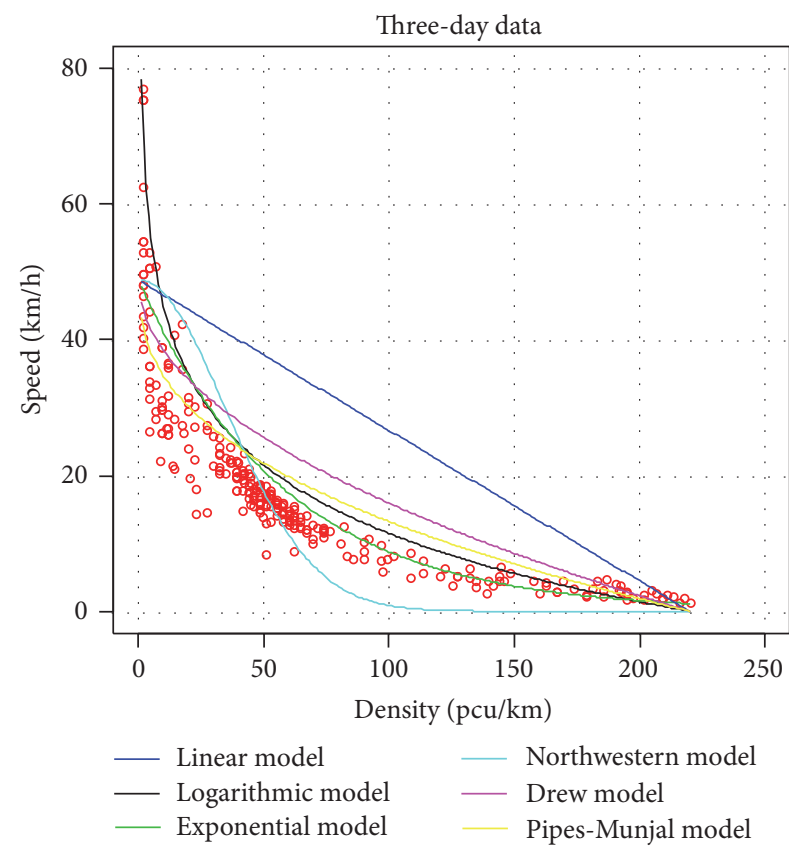

(b) Graph of three-day data

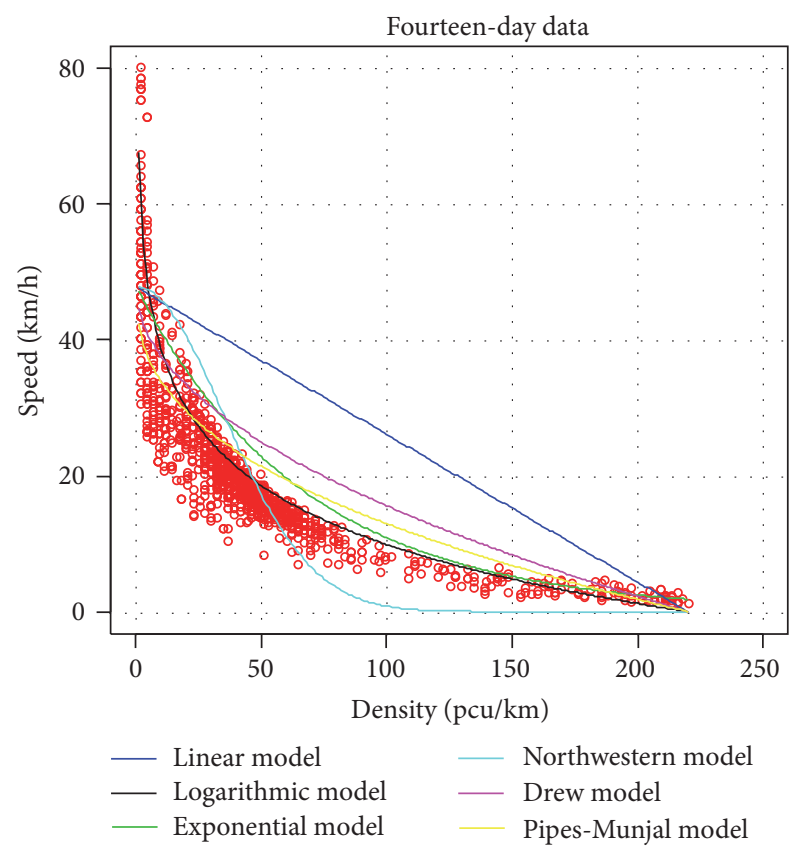

(d) Graph of fourteen-day data

FIgURe 2: Comparison of six speed-density models.

\section{The Description Method of the Speed- Density Model for Interrupted Traffic Flow}

3.1. The Characteristics of the Data of Interrupted Traffic. Coil data for one day, three days, seven days, and fourteen days were selected to compare and analyze the discontinuous flow data and the existing six speed-density models, as shown in Figure 2. We found the following:

(1) The six models' performance was poor when the coil data of interrupted traffic flow were fitted, illustrating that although suitable for uninterrupted traffic flow 


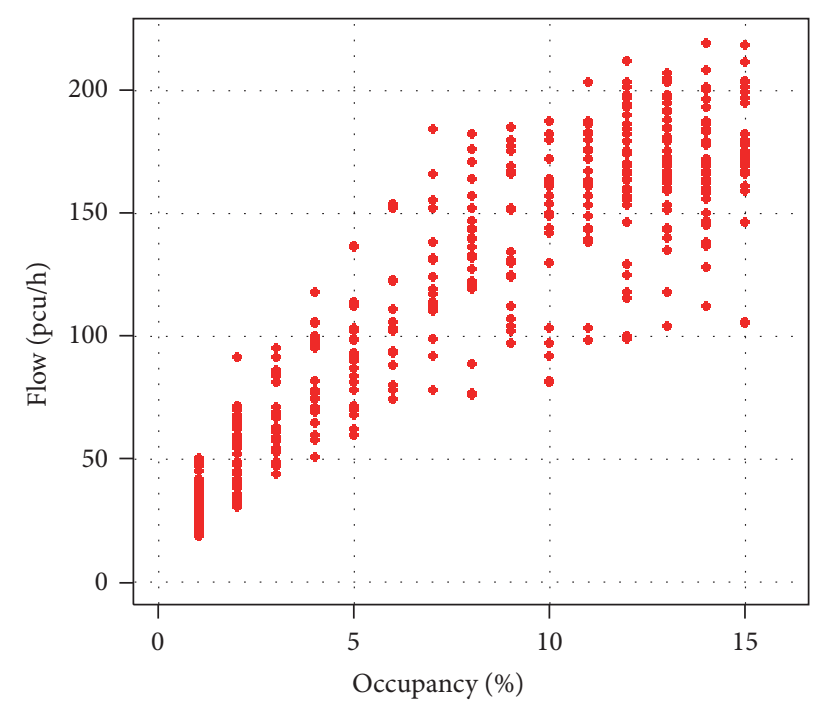

FIGURE 3: Flow-occupancy graph of small density.

they are unsuitable for describing the speed-density relation of interrupted traffic flow because of diverse data sources, different traffic environments, or other factors. In contrast, the logarithmic model gave the best performance and the linear model gave the worst performance.

(2) The interval value of critical densities $k_{m}$ of one-day, three-day, seven-day, and fourteen-day data sets was $[62.56 \mathrm{pcu} / \mathrm{km}, 71.23 \mathrm{pcu} / \mathrm{km}]$, and most of the data were located in $k<k_{m}$ range, meaning unimpeded flow data accounted for the absolute proportion, so the traffic flow of the location coil was in a state of flow most of the time.

(3) When $k<k_{m}$, with the increase of density, the velocity decreased sharply; when $k>k_{m}$, as the density increased, the velocity decreased slowly, and the speed variation amplitude was very small.

(4) When the density was small, the speed had a large range of values, of which the largest was $[23 \mathrm{~km} / \mathrm{h}$, $72 \mathrm{~km} / \mathrm{h}$ ]. We filtered out the small-density data to obtain a scatter diagram of flow and occupancy which were directly collected by a loop detector, as shown in Figure 3. In Figure 3 it is obvious that the loop detector acquires large-range flow values for the same occupancy value, and the largest range can reach $100 \mathrm{pcu} / \mathrm{h}$. Hence, after calculating speed and density by density formula and velocity formula, speed accordingly has a large range of values for the same density in speed-density diagram.

(5) In addition, the density values were found to be near a number of points, and the difference between adjacent points was approximately equal to a certain value.

From the above analysis, we found that, because of the big differences between uninterrupted and interrupted traffic flow, existing models suitable for uninterrupted traffic flow are unsuited for describing the speed-density relation of interrupted traffic flow. What is more, the flow collected by a loop detector has a large range of values. Therefore, for the speed-density relation of interrupted traffic flow, we must find a new descriptive method.

3.2. Description Method of Speed-Density Relationship for Interrupted Traffic Flow. Because of the difference between the uninterrupted and interrupted traffic flow and the volatility of speed, the speed-density relationship cannot be adequately described by a single model, so we use two curves, $u^{\text {upper }}$ and $u^{\text {lower }}$, to describe the supremum and infimum of velocity values:

$$
\begin{aligned}
& u^{\text {upper }}=g^{\text {upper }}\left(U^{\text {upper }}\right), \\
& u^{\text {lower }}=g^{\text {lower }}\left(U^{\text {lower }}\right),
\end{aligned}
$$

where $U^{\text {upper }}$ and $U^{\text {lower }}$ are, respectively, the upper and lower bounds of velocity and $g^{\text {upper }}$ and $g^{\text {lower }}$ are fitting functions.

Divide the density interval $\left[k_{\min }, k_{\max }\right]$ into $n$ connected intervals $k_{1}, k_{2}, \ldots, k_{n}$. Partition data $D$ as $D_{1}, D_{2}, \ldots, D_{n}$ by density intervals, and correspondingly get speed sets $U_{1}, U_{2}, \ldots, U_{n}$, causing that, for any $i \in(1,2, \ldots, n)$, we have

$$
W\left(U_{i}\right)>W_{\alpha},
$$

where $W\left(U_{i}\right)$ is used test for $U_{i}$ with the Shapiro-Wilk normal test method. Sort $m$ independent observations in $U_{i}$ by nondescending order, recorded as $x_{1}, x_{2}, \ldots, x_{m}$, and construct the $W$-test statistic

$$
W=\frac{\left[\sum_{i=1}^{m} a_{i} \times\left(x_{m+1-i}-x_{i}\right)\right]^{2}}{\sum_{i=1}^{m} a_{i} \times\left(x_{i}-\bar{x}\right)^{2}},
$$

where $a_{i}$ is the coefficient when sample size is $m$. When the population distribution is normal distribution, the value of $W$ should be close to one. $\alpha$ quantile $W_{\alpha}$ of statistic $W$ can be obtained by the look-up table method. When $W \leq W_{\alpha}$, the original hypothesis should be rejected at the significant level, indicating that $U_{i}$ does not obey normal distribution; when $W>W_{\alpha}$, the original hypothesis cannot be rejected, and $U_{i}$ satisfies normal distribution.

Under the conditions of (8), for every $i \in(1,2, \ldots, n)$, extract the upper quantile $u_{i}^{\text {upper }}$ and lower quantile $u_{i}^{\text {lower }}$ as the upper and lower critical values of speed for density interval $k_{i}$.

$$
\begin{aligned}
& u_{i}^{\text {upper }}=\text { qnorm }\left(\text { upper, mean }\left(U_{i}\right), \operatorname{sd}\left(U_{i}\right)\right), \\
& u_{i}^{\text {lower }}=\text { qnorm }\left(\text { lower, mean }\left(U_{i}\right), \operatorname{sd}\left(U_{i}\right)\right),
\end{aligned}
$$

where qnorm( ) is quantile function, mean( ) calculates the mean value of $U_{i}$, and $\operatorname{sd}()$ calculates the variance of $U_{i}$.

Get the upper bound and lower bound sets

$$
\begin{aligned}
U^{\text {upper }} & =\left\{u_{i}^{\text {upper }}, i=1,2, \ldots, n\right\}, \\
U^{\text {lower }} & =\left\{u_{i}^{\text {lower }}, i=1,2, \ldots, n\right\} .
\end{aligned}
$$

Fit $U^{\text {upper }}$ and $U^{\text {lower }}$ using the nonlinear least square method. The tabulated function $u_{i}=u\left(k_{i}\right), i=1,2, \ldots, n$, is 
available by (10). Then we need to obtain the fitting function, $g(k)=a_{0}+a_{1} \times g_{1}(k)+\cdots+a_{p} \times g_{p}(k)$, making the sum of squared deviations

$$
\begin{aligned}
S & \left(a_{0}, a_{1}, \ldots, a_{p}\right)=\sum_{i=1}^{n}\left[g\left(k_{i}\right)-u_{i}\right]^{2} \\
& =\sum_{i=1}^{n}\left[a_{0}+a_{1} \times g_{1}\left(k_{i}\right)+\cdots+a_{p} \times g_{p}\left(k_{i}\right)-u_{i}\right]^{2} .
\end{aligned}
$$

Take the minimum, of which $g_{1}(k), g_{2}(k), \ldots, g_{p}(k)$ are $p$ nonmergeable monomials of variable $k$, and $a_{0}, a_{1}, \ldots, a_{p}$ are the coefficients of monomials. $S$ is a nonnegative polynomial of $a_{0}, a_{1}, \ldots, a_{p}$, so there must be a minimum value. Respectively, calculate partial derivatives of $S$ for $a_{0}, a_{1}, \ldots, a_{p}$, and make them equal to zero.

$$
\frac{\partial S}{\partial a_{i}}=0, \quad i=0,1, \ldots, p .
$$

Equation (13) is expanded as follows:

$$
\begin{aligned}
& \frac{\partial S}{\partial a_{0}}=2 \\
& \quad \times \sum_{i=1}^{n}\left[a_{0}+a_{1} \times g_{1}\left(k_{i}\right)+\cdots+a_{p} \times g_{p}\left(k_{i}\right)-u_{i}\right] \\
& \quad=0, \\
& \frac{\partial S}{\partial a_{1}}=2 \\
& \quad \times \sum_{i=1}^{n}\left[a_{0}+a_{1} \times g_{1}\left(k_{i}\right)+\cdots+a_{p} \times g_{p}\left(k_{i}\right)-u_{i}\right] \\
& \quad \times g_{1}\left(k_{i}\right)=0, \\
& \frac{\partial S}{\partial a_{p}}=2 \\
& \quad \times \sum_{i=1}^{n}\left[a_{0}+a_{1} \times g_{1}\left(k_{i}\right)+\cdots+a_{p} \times g_{p}\left(k_{i}\right)-u_{i}\right] \\
& \quad \times g_{p}\left(k_{i}\right)=0 .
\end{aligned}
$$

Continue to expand (14):

$$
\begin{gathered}
a_{0} \times n+a_{1} \times \sum_{i=1}^{n} g_{1}\left(k_{i}\right)+\cdots+a_{p} \times \sum_{i=1}^{n} g_{p}\left(k_{i}\right)=\sum_{i=1}^{n} u_{i}, \\
a_{0} \times \sum_{i=1}^{n} g_{1}\left(k_{i}\right)+a_{1} \times \sum_{i=1}^{n}\left[g_{1}\left(k_{i}\right)\right]^{2}+\cdots+a_{p} \\
\quad \times \sum_{i=1}^{n}\left[g_{p}\left(k_{i}\right) \times g_{1}\left(k_{i}\right)\right]=\sum_{i=1}^{n}\left[u_{i} \times g_{1}\left(k_{i}\right)\right],
\end{gathered}
$$

$$
\begin{aligned}
a_{0} & \times \sum_{i=1}^{n} g_{p}\left(k_{i}\right)+a_{1} \times \sum_{i=1}^{n}\left(g_{1}\left(k_{i}\right) \times g_{p}\left(k_{i}\right)\right)+\cdots+a_{p} \\
& \times \sum_{i=1}^{n}\left[g_{p}\left(k_{i}\right)\right]^{2}=\sum_{i=1}^{n}\left[u_{i} \times g_{p}\left(k_{i}\right)\right]
\end{aligned}
$$

and get its matrix form

$$
\begin{aligned}
& {\left[\begin{array}{cccc}
n & \sum_{i=1}^{n} g_{1}\left(k_{i}\right) & \ldots & \sum_{i=1}^{n} g_{p}\left(k_{i}\right) \\
\sum_{i=1}^{n} g_{1}\left(k_{i}\right) & \sum_{i=1}^{n}\left[g_{1}\left(k_{i}\right)\right]^{2} & \cdots & \sum_{i=1}^{n}\left[g_{p}\left(k_{i}\right) \times g_{1}\left(k_{i}\right)\right] \\
\vdots & \vdots & \vdots & \vdots \\
\sum_{i=1}^{n} g_{p}\left(k_{i}\right) & \sum_{i=1}^{n}\left[g_{1}\left(k_{i}\right) \times g_{p}\left(k_{i}\right)\right] & \cdots & \sum_{i=1}^{n}\left[g_{p}\left(k_{i}\right)\right]^{2}
\end{array}\right]} \\
& \times\left[\begin{array}{c}
a_{0} \\
a_{1} \\
\vdots \\
a_{p}
\end{array}\right]=\left[\begin{array}{c}
\sum_{i=1}^{n} u_{i} \\
\sum_{i=1}^{n}\left[u_{i} \times g_{1}\left(k_{i}\right)\right] \\
\vdots \\
\sum_{i=1}^{n}\left[u_{i} \times g_{p}\left(k_{i}\right)\right]
\end{array}\right] .
\end{aligned}
$$

Solve (16), and $a_{0}, a_{1}, \ldots, a_{p}$ are available.

Using the above least square method to fit $U^{\text {upper }}$ and $U^{\text {lower }}$, respectively, obtains

$$
\begin{aligned}
& u^{\text {upper }}(k)=g^{\text {upper }}\left(k, a_{0}, a_{1}, \ldots, a_{p}\right), \\
& u^{\text {lower }}(k)=g^{\text {lower }}\left(k, a_{0}, a_{1}, \ldots, a_{p}\right)
\end{aligned}
$$

and upper and lower curves $u^{\text {upper }}(k)$ and $u^{\text {lower }}(k)$, which is the speed-density relation description model.

\section{Experiment and Analysis}

The experiment data is collected by the coil detectors underground closed to Optical Valley Walking Street in Wuhan, China. Coil detectors collect data every 15 minutes, recording time, flow, occupancy, and so forth, as shown in Table 2.

Use the method in $[20,21]$ to calculate speed and density, and the ratio of the amount of data between two model curves to the total amount of experiment data is used to describe the performance of model. The loop detector in the outer lane measures the traffic flow of straight and right-turning lanes, and the loop detector in the inner lane measures the traffic flow of the left-turning lane. The traffic flow characteristics of two loop detectors must have certain differences. Therefore, analyze the coil data of both the outer lane and the inner lane to find the diversity of their speed-density relationship.

4.1. Coil Data Analysis of the Outer Lane. The experimental steps are as follows.

Step 1. Analyze coil data of the outer lane and find that density values are clustered at a number of points $k_{1}, k_{2}, \ldots, k_{n}$, 
TABLE 2: The data example.

\begin{tabular}{lccccc}
\hline Date & Week & Flow & Occupancy & Minute & id \\
\hline $2014 / 11 / 20$ & 4 & 132 & 7 & $0: 00: 00$ & 41751051 \\
$2014 / 11 / 20$ & 4 & 91 & 5 & $0: 15: 00$ & 41751051 \\
$2014 / 11 / 20$ & 4 & 98 & 7 & $0: 30: 01$ & 41751051 \\
$2014 / 11 / 20$ & 4 & 103 & 6 & $0: 45: 01$ & 41751051 \\
$2014 / 11 / 20$ & 4 & 77 & 4 & $1: 00: 00$ & 41751051 \\
$2014 / 11 / 20$ & 4 & 64 & 75 & $1: 15: 00$ & 41751051 \\
$2014 / 11 / 20$ & 4 & 40 & & $1: 40: 01$ & 41751051 \\
$2014 / 11 / 20$ & 4 & & & & 41751051 \\
\hline
\end{tabular}

where the mean value of the difference between the adjacent points is about $2.5 \mathrm{pcu} / \mathrm{km}$. Divide density $k$ into a number of intervals with length $2.5 \mathrm{pcu} / \mathrm{km}$ by $k_{1}, k_{2}, \ldots, k_{n}$.

Step 2. Correspondingly split data $D$ into small data sets $D_{1}, D_{2}, \ldots, D_{n}$ according to density segmentations, and get data sets of speed $U_{1}, U_{2}, \ldots, U_{n}$.

Step 3. Execute a distribution test for $U_{i}$ where the result shows that one data set is too small to meet the requirements of the test. Merge the adjacent density segments in Step 2 to enlarge the amount of the small data set. Redo the distribution test for the new data set, more than $80 \%$ of which meets the normal distribution, with totally $95 \%$ of the total data satisfying the normal distribution, which makes it reasonable to consider all the small data set satisfying the normal distribution.

Step 4. Get two quantiles $u_{i}^{\text {upper }}$ and $u_{i}^{\text {lower }}$ of speed set $U_{i}$ as upper and lower critical values of velocity for density $k_{i}$.

Step 5. Then have upper and lower critical value set $U^{\text {upper }}=$ $\sum_{i=1}^{n} u_{i}^{\text {upper }}$ and $U^{\text {lower }}=\sum_{i=1}^{n} u_{i}^{\text {lower }}$.

Step 6 (fit $u^{\text {upper }}$ and $u^{\text {lower }}$ ). Because the loop detector is located near commercial street which has heavy traffic, we use the logarithmic model to formulize the data.

$$
\begin{aligned}
& u^{0.95}=14.204 \times \ln \left(\frac{216.412}{k}\right), \\
& u^{0.05}=7.169 \times \ln \left(\frac{254.497}{k}\right) .
\end{aligned}
$$

Figure 4 shows the validation result of the speed-density logarithmic model of the outer lane when upper value = 0.95 and lower value $=0.05$. Equations (18) correspondingly are the green and blue curves in Figure 4, which is the speed-density model of interrupted traffic flow created by the new description method. Significant test results indicate that $P$ values of two regression coefficients of two curves are minima $(P<2 e-16)$, which means that coefficients are significant and two log models constructed with density as the independent variable are applied to estimate velocity as the dependent variable.

The coil data of the outer lane for two weeks, four weeks, six weeks, and eight weeks are, respectively, selected

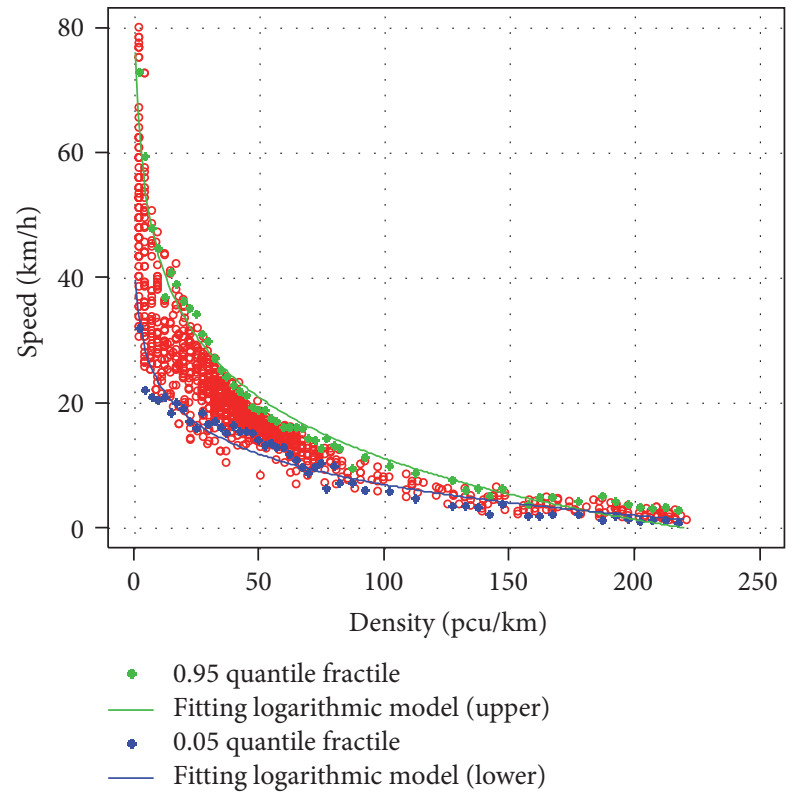

FIGURE 4: Speed-density logarithmic model of the outside lane.

and four groups of parameters are established for model validation. Table 3 gives the ratio of the data between two logarithmic curves to the total amount of data in each case. Make a longitudinal observation; it is obvious that, with upper value increasing and lower value decreasing, the proportion increases accordingly, where amplitudes are obvious, respectively, $7.2 \%, 6.2 \%$, and $6.9 \%$. On the other hand, the main transverse trend is that the proportion increases along with the increase of experiment data loosely, where, however, sixweek data has the best performance. The above suggests that the two logarithmic models are able to describe the speeddensity relation of the outer lane. Figure 5 shows the four groups' validation results when upper value $=0.95$ and lower value $=0.05$.

4.2. Coil Data Analysis of the Inner Lane. We select coil data of the inner lane and follow Steps 1 to 5 as for the outer lane. When fitting sets $u^{\text {upper }}$ and $u^{\text {lower }}$ at Step 6, we find that the speed-density models proposed by scholars all have poor performance with goodness of fit of less than 0.5 , 
TABLE 3: Validation results of the model of the outer lane.

\begin{tabular}{|c|c|c|c|c|c|}
\hline Parameters & Two-week data & Four-week data & Six-week data & Eight-week data & Average \\
\hline $\begin{array}{l}\text { Upper value }=0.80 \\
\text { Lower value }=0.20\end{array}$ & $61.9 \%$ & $65.8 \%$ & $66.2 \%$ & $66.1 \%$ & $65.0 \%$ \\
\hline $\begin{array}{l}\text { Upper value }=0.85 \\
\text { Lower value }=0.15\end{array}$ & $68.9 \%$ & $73.1 \%$ & $73.6 \%$ & $73.3 \%$ & $72.2 \%$ \\
\hline $\begin{array}{l}\text { Upper value }=0.90 \\
\text { Lower value }=0.10\end{array}$ & $74.5 \%$ & $78.8 \%$ & $80.4 \%$ & $79.7 \%$ & $78.4 \%$ \\
\hline $\begin{array}{l}\text { Upper value }=0.95 \\
\text { Lower value }=0.05\end{array}$ & $84.2 \%$ & $85.1 \%$ & $86.7 \%$ & $85.3 \%$ & $85.3 \%$ \\
\hline
\end{tabular}

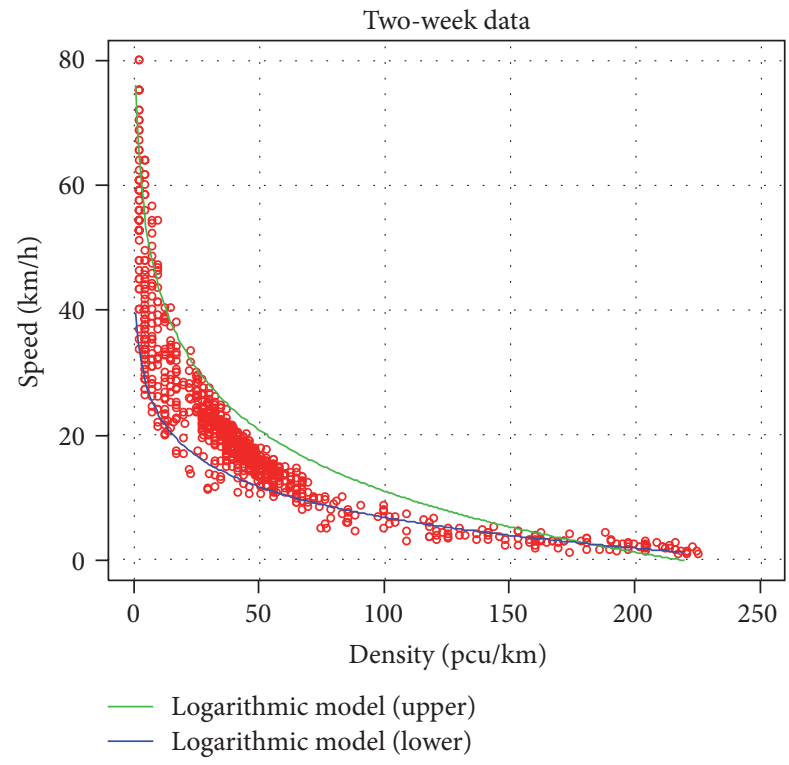

(a) Validation result for two-week data

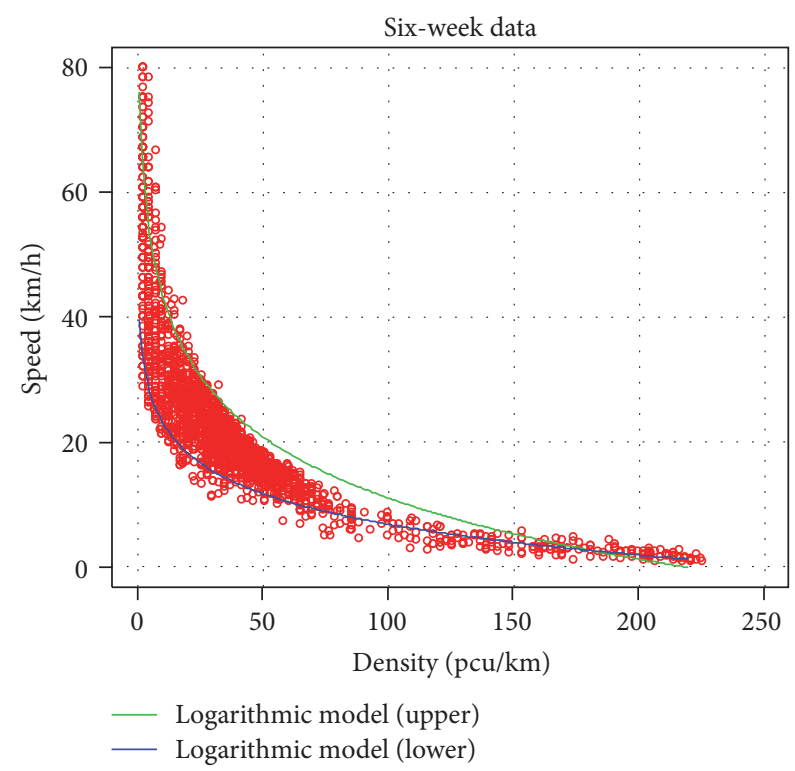

(c) Validation result for six-week data

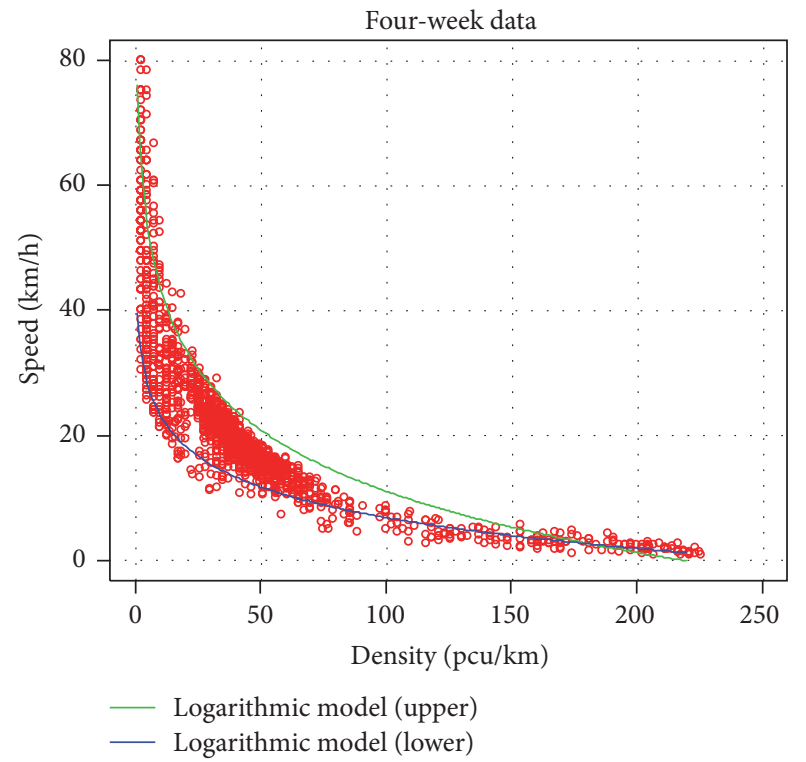

(b) Validation result for four-week data

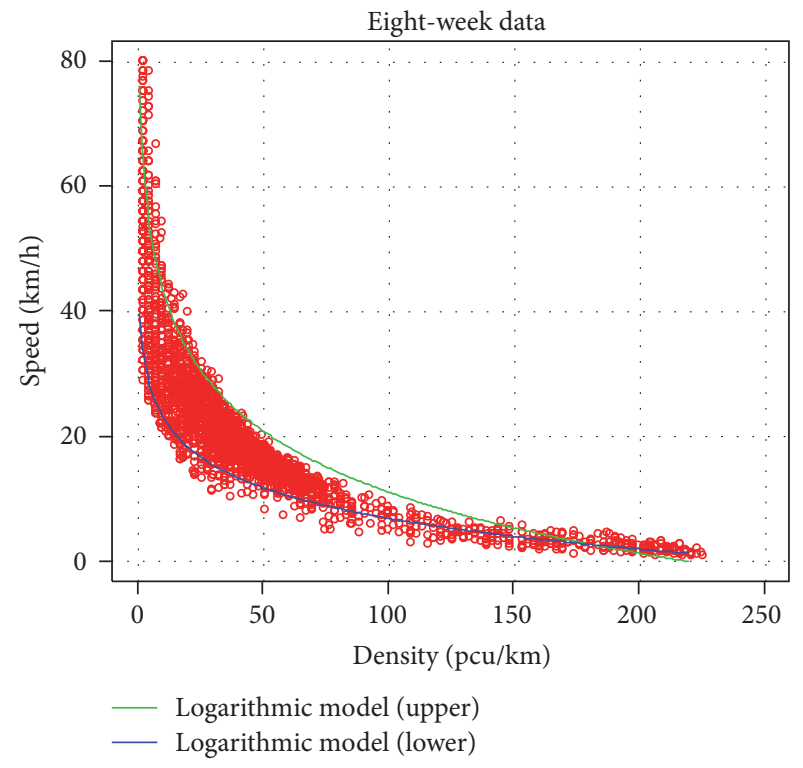

(d) Validation result for eight-week data

FIGURE 5: Validation result of the speed-density logarithmic model of the outside lane. 
TABLE 4: Validation results of the model of the inner lane.

\begin{tabular}{|c|c|c|c|c|c|}
\hline Parameters & Two-week data & Four-week data & Six-week data & Eight-week data & Average \\
\hline $\begin{array}{l}\text { Upper value }=0.80 \\
\text { Lower value }=0.20\end{array}$ & $80.0 \%$ & $77.9 \%$ & $81.3 \%$ & $78.8 \%$ & $79.5 \%$ \\
\hline $\begin{array}{l}\text { Upper value }=0.85 \\
\text { Lower value }=0.15\end{array}$ & $82.0 \%$ & $82.3 \%$ & $85.2 \%$ & $83.1 \%$ & $83.2 \%$ \\
\hline $\begin{array}{l}\text { Upper value }=0.90 \\
\text { Lower value }=0.10\end{array}$ & $83.8 \%$ & $84.9 \%$ & $86.4 \%$ & $85.3 \%$ & $85.1 \%$ \\
\hline $\begin{array}{l}\text { Upper value }=0.95 \\
\text { Lower value }=0.05\end{array}$ & $89.0 \%$ & $90.3 \%$ & $88.9 \%$ & $89.8 \%$ & $89.5 \%$ \\
\hline
\end{tabular}

which suggests that a single model cannot accurately describe the quantile set of the coil data. Thus we consider using a segmentation model.

In the density-flow curve there is a critical density $k_{m}$, which is the density of maximum traffic flow, as shown in Figure 1 . When the density $k<k_{m}$, the traffic is in a state of flow; when $k>k_{m}$, the traffic flow gradually becomes crowded. Therefore, consider using $k_{m}$ as the critical value of the subsection.

A density-flow curve is obtained by local polynomial regression fitting, and the density value at the curve vertex is just $k_{m}$. Take $k_{m}$ as the critical value and piecewise analyze $u_{k<k_{m}}^{\text {upper }}, u_{k<k_{m}}^{\text {lower }}, u_{k>k_{m}}^{\text {upper }}$, and $u_{k>k_{m}}^{\text {lower }}$. The analysis shows that the quantile set $u_{k<k_{m}}^{\text {upper }}$ and $u_{k<k_{m}}^{\text {lower }}$ agrees with the exponential model, and the quantile set $u_{k>k_{m}}^{\text {upper }}$ and $u_{k>k_{m}}^{\text {lower }}$ has good agreement with the logarithmic model.

$$
\begin{aligned}
& u^{0.95}= \begin{cases}69.647 \times e^{-k / 14.449}+12.716, & k<k_{m} \\
8.227 \times \ln \left(\frac{254.971}{k}\right), & k \geq k_{m},\end{cases} \\
& u^{0.05}= \begin{cases}51.08 \times e^{-k / 100.10}-22.45, & k<k_{m} \\
5.337 \times \ln \left(\frac{243.306}{k}\right), & k \geq k_{m} .\end{cases}
\end{aligned}
$$

Figure 6 shows the fitting result of a segmentation model of the outer lane when upper value $=0.95$ and lower value $=0.05$, and (19) are the models corresponding to the green curve and blue curve in Figure 6, which is the speed-density model of interrupted traffic flow via the new description method. $P$ value of each parameter is very small, suggesting the coefficient is very significant.

The coil data of the inner lane for two weeks, four weeks, six weeks, and eight weeks are, respectively, selected and four groups of parameters are established for the model validation, the same as that for the outer lane. Table 4 gives the ratio of the data between two logarithmic curves to the total amount of data in each case. Comparing the result with that of the outer lane, we find that the validation results of the model of the inner lane are better with greater ratio.

Take a longitudinal observation; similarly, it is obvious that with upper value increasing and lower value decreasing, the proportion increases accordingly, where amplitudes are smaller than that of outer lane, respectively $3.7 \%, 1.9 \%$, and $4.3 \%$. The main transverse trend is the same as outer lane

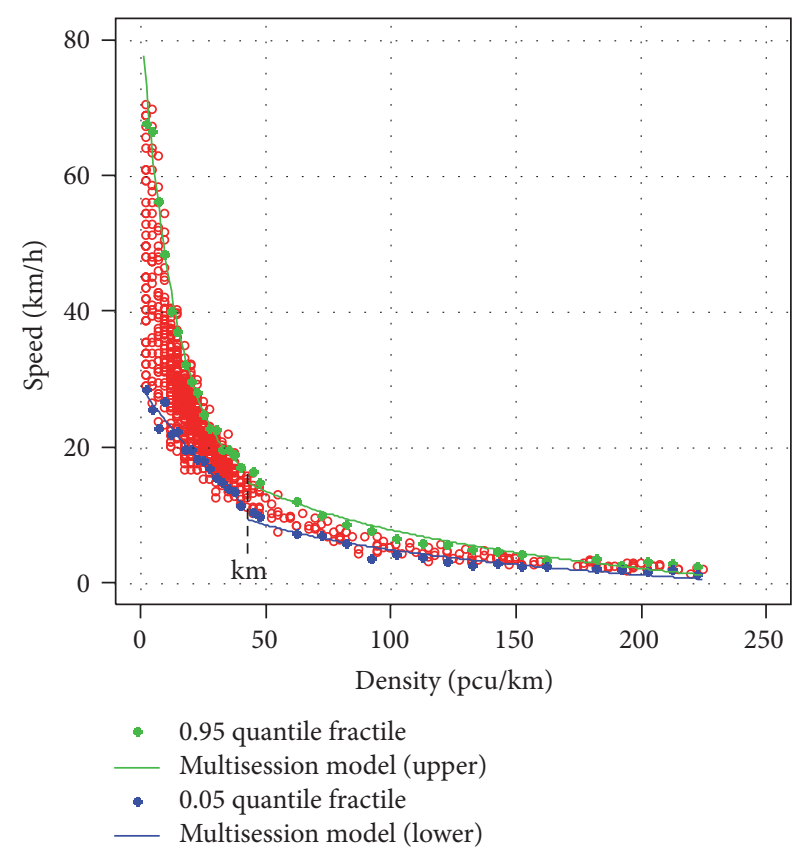

FIGURE 6: Speed-density multisession model of the inside lane.

except the case of upper value $=0.95$ and lower value $=0.05$. The result indicates that the two segmentation models are suitable for describing the speed-density relation of the inner lane. Figure 7 shows the four groups' validation results when upper value $=0.95$ and lower value $=0.05$.

\subsection{Experimental Result Analysis}

4.3.1. Difference between the Models of the Outer Lane and the Inner Lane. The loop detector of the outer lane measures right-turning and straight lanes, and the coil is located in a road adjacent to a commercial pedestrian street with a heavy flow of people and traffic. A logarithmic model is applied to describe traffic flow with large density, and therefore it is accepted that the coil data of the outer lane satisfy the logarithmic model.

The loop detector of the inner lane measures the leftturning lane which also has heavy traffic flow. The speeddensity relation of the inner lane does not satisfy the single log model but is suitable for the segmentation model. The 


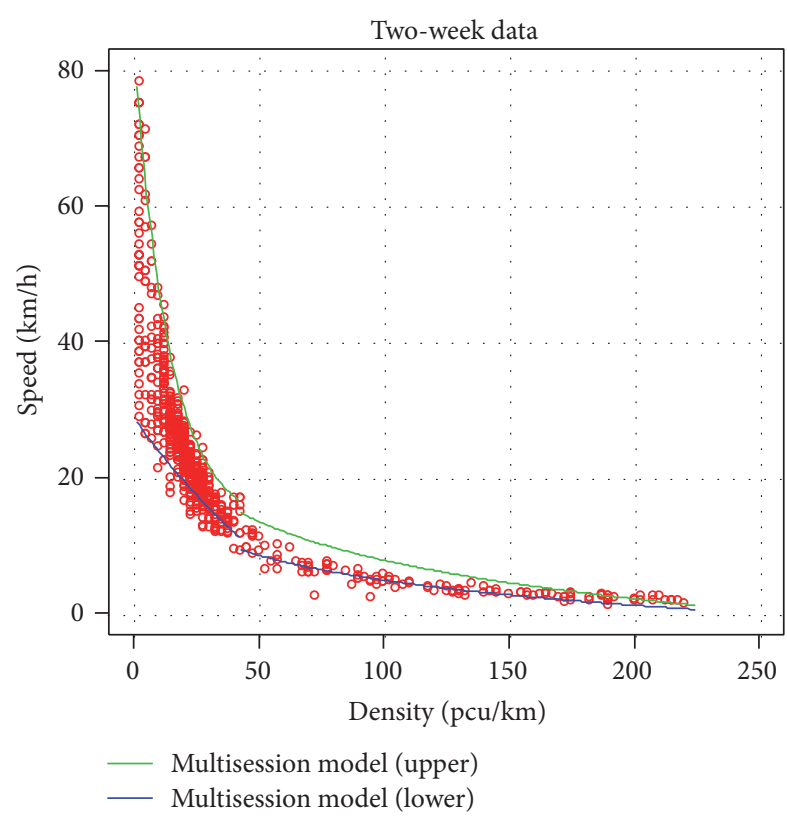

(a) Validation result for two-week data

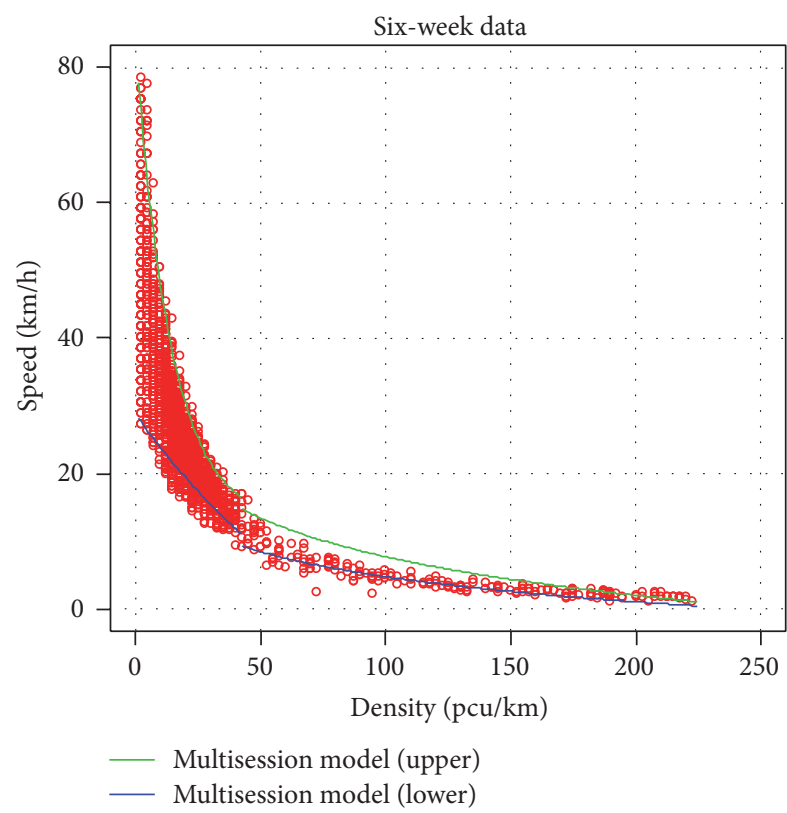

(c) Validation result for six-week data

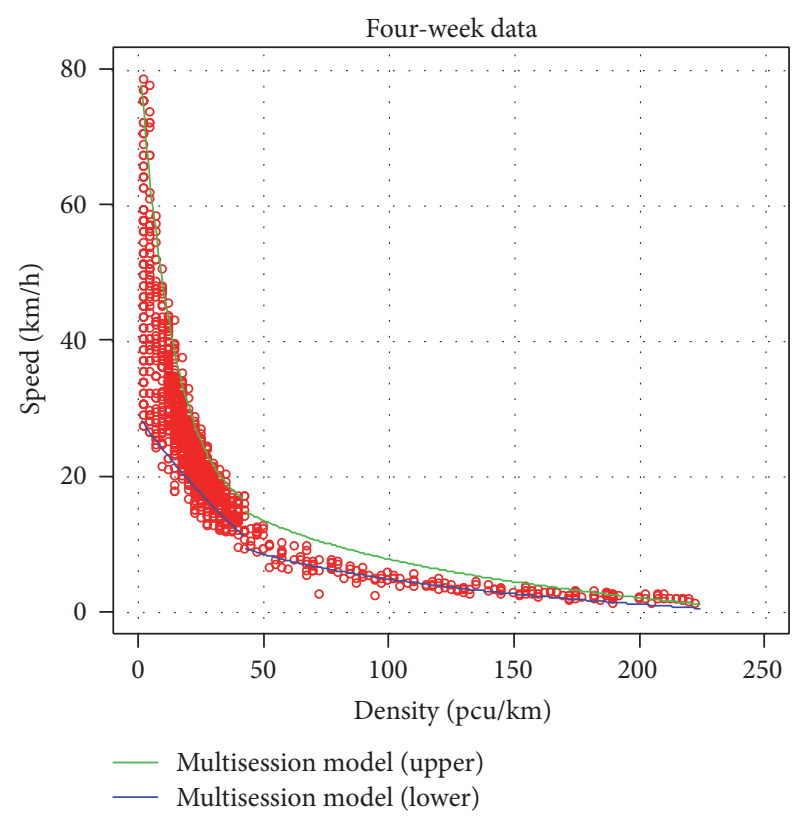

(b) Validation result for four-week data

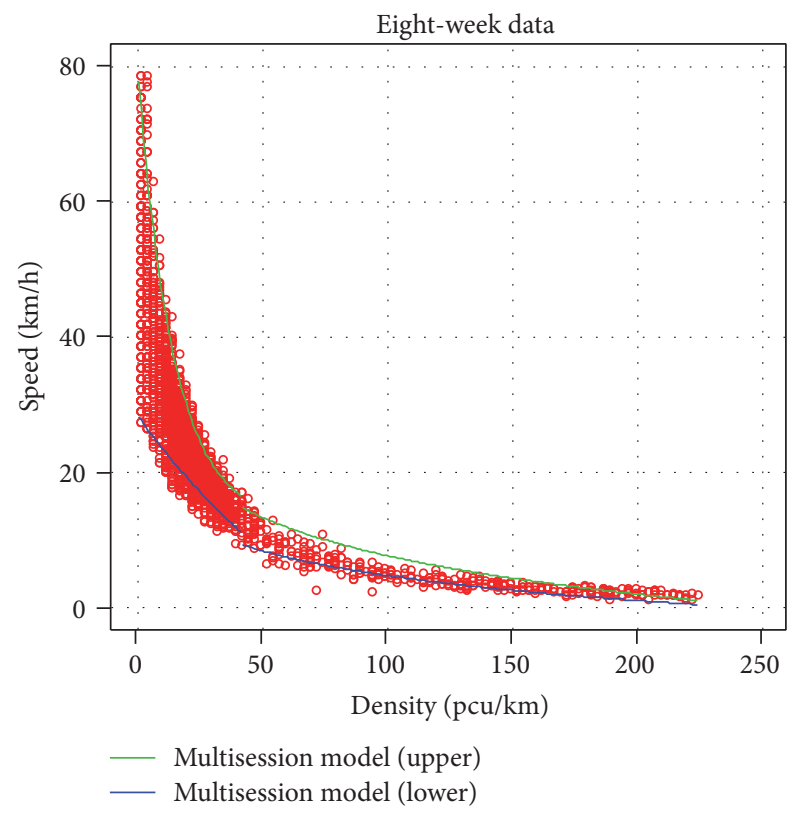

(d) Validation result for eight-week data

FIGURE 7: The validation result of the speed-density multisession model of the inside lane.

exponential models of (19) are two-item types with intercepts, instead of Underwood's monomial exponential model. They suggest that the traffic flow of the inner lane differs from the traffic flow of the freeway and outer lane.

4.3.2. Characteristic Analysis of Traffic Flow. The critical density $k_{m}$ of the outer lane in Figure 6 is $53.6 \mathrm{pcu} / \mathrm{km}$, and most of the density values are less than $k_{m}$ or in a small range of $k_{m}$; similarly, for the inner lane, most of the density values are smaller than $k_{m}$, and the data in the range $k>k_{m}$ are sparse. This illustrates that (1) most of the time the road segment where loop detectors located is unblocked, where the data with big density values which may lead to congestion is just a small proportion, and, (2) compared with the outer lane, the proportion of the density $k<k_{m}$ of the inner lane is greater, illustrating that the inner lane is more unimpeded than the outer lane.

In summary, the new description method can satisfactorily describe the speed-density relation of interrupted traffic, where the speed-density relation of the outer lane meets the 
logarithmic model and the inner lane meets the segment model. What is more, the road segment where loop detectors located is unblocked at most of the time; the inner lane is more unimpeded than the outer lane.

\section{Conclusion}

In this paper, the characteristics of urban interrupted flow data were analyzed, and it was found that they differ from the data of uninterrupted flow. Since the existing classical models cannot describe them very well, a description method of speed-density relation for interrupted traffic flow was proposed where the upper and lower curves were used as the upper and lower bounds of the predicted speed. In this method, the speed was divided into small data sets which satisfied the normal distribution, and two quantiles of normal distribution were obtained as the predicted values. Then two quantile sets were fitted to get two curves as the speed-density relation model of the interrupted traffic flow. Finally, the coil data of the outer and inner lanes were applied for model validation. The results showed that the new method can give a good description of the speed-density relationship of interrupted traffic flow and get different model results for the outer lane and inner lane, whereby the speed-density relation of the outer lane satisfies the logarithmic model and the inner lane satisfies the segment model instead of the single model, where when the density is less than critical density, it conforms to the exponential model and otherwise the logarithmic model. The fitting results of the internal and external lanes were analyzed in combination with the actual local road environment and traffic flow theory. So this model can provide favorable data analysis and presentation for city traffic, thus to provide decision support for intelligent transportation.

\section{Competing Interests}

The authors declare that they have no competing interests.

\section{Acknowledgments}

The work is partly supported by NSFC (no. 61472149), the Fundamental Research Funds for the Central Universities (2015QN67), the Wuhan Youth Science and Technology Plan (2016070204010132), and the National $863 \mathrm{Hi}$-Tech Research and Development Program under Grant 2015AA01A203.

\section{References}

[1] D. B. Greenshields, R. J. Biddins, S. W. Channing et al., "A study in highway capacity," Highway Research Board Proceedings, vol. 14, no. 1, pp. 448-477, 1935.

[2] H. Z. Wang, D. h. Ni, Q.-Y. Chen, and J. Li, "Stochastic modeling of the equilibrium speed-density relationship," Journal of Advanced Transportation, vol. 47, no. 1, pp. 126-150, 2013.

[3] A. K. Gupta, S. Sharma, and P. Redhu, "Analyses of lattice traffic flow model on a gradient highway," Communications in Theoretical Physics, vol. 62, no. 3, pp. 393-404, 2014.
[4] B. G. Heydecker and J. D. Addison, "Analysis and modelling of traffic flow under variable speed limits," Transportation Research Part C: Emerging Technologies, vol. 19, no. 2, pp. 206-217, 2011.

[5] X.-L. Ma, D.-F. Ma, D.-H. Wang, and S. Lin, "Modeling of speeddensity relationship in traffic flow based on logistic curve," China Journal of Highway and Transport, vol. 28, no. 4, pp. 94100, 2015.

[6] C.-F. Shao, C.-Z. Xiao, B.-B. Wang, and M. Meng, "Speeddensity relation model of congested traffic flow under minimum safety distance constraint," Journal of Traffic and Transportation Engineering, vol. 15, no. 1, pp. 92-99, 2015.

[7] H. Z. Wang, J. Li, Q.-Y. Chen, and D. Ni, "Logistic modeling of the equilibrium speed-density relationship," Transportation Research Part A: Policy and Practice, vol. 45, no. 6, pp. 554-566, 2011.

[8] V. Corcoba Magaña and M. Muñoz-Organero, "WATI: warning of traffic incidents for fuel saving," Mobile Information Systems, vol. 2016, Article ID 3091516, 16 pages, 2016.

[9] M.-W. Li, W.-C. Hong, and H.-G. Kang, "Urban traffic flow forecasting using Gauss-SVR with cat mapping, cloud model and PSO hybrid algorithm," Neurocomputing, vol. 99, no. 1, pp. 230-240, 2013.

[10] F. Ahmad, I. Khan, S. A. Mahmud et al., "Real time evaluation of shortest remaining processing time based schedulers for traffic congestion control using wireless sensor networks," in Proceedings of the International Conference on Connected Vehicles and Expo (ICCVE '13), pp. 381-391, Las Vegas, Nev, USA, 2013.

[11] H. Y. Shang and Y. Peng, "A new cellular automaton model for traffic flow considering realistic turn signal effect," Science China Technological Sciences, vol. 55, no. 6, pp. 1624-1630, 2012.

[12] K. Jung, M. Do, J. Lee, and Y. Lee, "Vehicle running characteristics for interrupted traffic flow by using cellular automata," The Journal of The Korea Institute of Intelligent Transport Systems, vol. 11, no. 6, pp. 31-39, 2012.

[13] J. M. del Castillo, "Three new models for the flow-density relationship: derivation and testing for freeway and urban data," Transportmetrica, vol. 8, no. 6, pp. 443-465, 2012.

[14] T.-Q. Tang, L. Caccetta, Y.-H. Wu, H.-J. Huang, and X.-B. Yang, "A macro model for traffic flow on road networks with varying road conditions," Journal of Advanced Transportation, vol. 48, no. 4, pp. 304-317, 2014.

[15] X. B. Yang, Z. Y. Gao, H. W. Guo, and M. Huan, "Survival analysis of car travel time near a bus stop in developing countries," Science China Technological Sciences, vol. 55, no. 8, pp. 2355-2361, 2012.

[16] R. Akçelik, "Relating flow, density, speed and travel time models for uninterrupted and interrupted traffic," Traffic Engineering and Control, vol. 37, no. 9, pp. 511-516, 1996.

[17] R. Jiang and Q.-S. Wu, "The traffic flow controlled by the traffic lights in the speed gradient continuum model," Physica A: Statistical Mechanics and Its Applications, vol. 355, no. 2-4, pp. 551-564, 2005.

[18] F. J. Wang, W. Wei, H. S. Qi et al., "Research on discontinuous flow speed-density relation model," Journal of Highway and Transportation Research and Development, vol. 31, no. 7, pp. 108114, 2014.

[19] H. Wang, D. Ni, Q.-Y. Chen, and J. Li, "Stochastic modeling of the equilibrium speed-density relationship," Journal of Advanced Transportation, vol. 47, no. 1, pp. 126-150, 2013. 
[20] F. Soriguera and F. Robusté, "Estimation of traffic stream space mean speed from time aggregations of double loop detector data," Transportation Research Part C: Emerging Technologies, vol. 19, no. 1, pp. 115-129, 2011.

[21] Y. Lao, G. Zhang, J. Corey, and Y. Wang, "Gaussian mixture model-based speed estimation and vehicle classification using single-loop measurements," Journal of Intelligent Transportation Systems: Technology, Planning, and Operations, vol. 16, no. 4, pp. 184-196, 2012. 

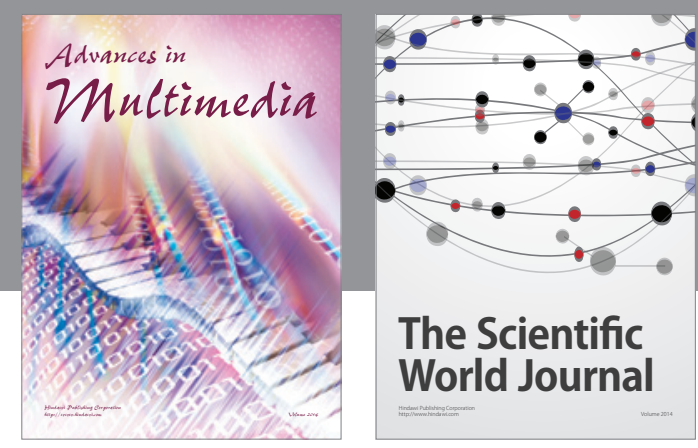

The Scientific World Journal
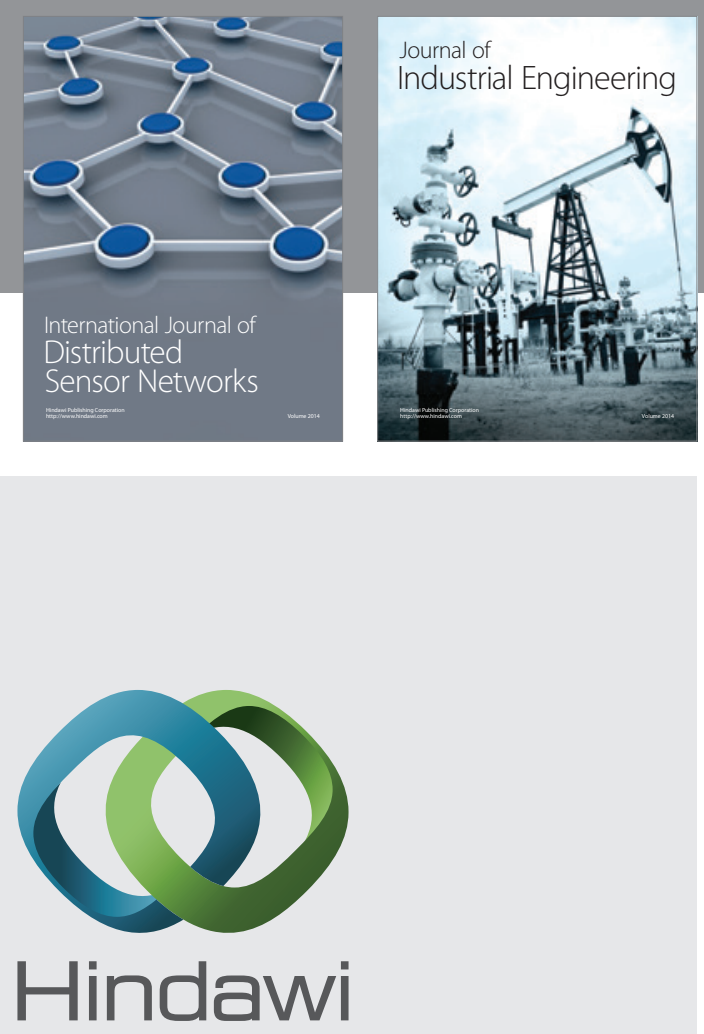

Submit your manuscripts at

http://www.hindawi.com

\section{Computer Networks} and Communications
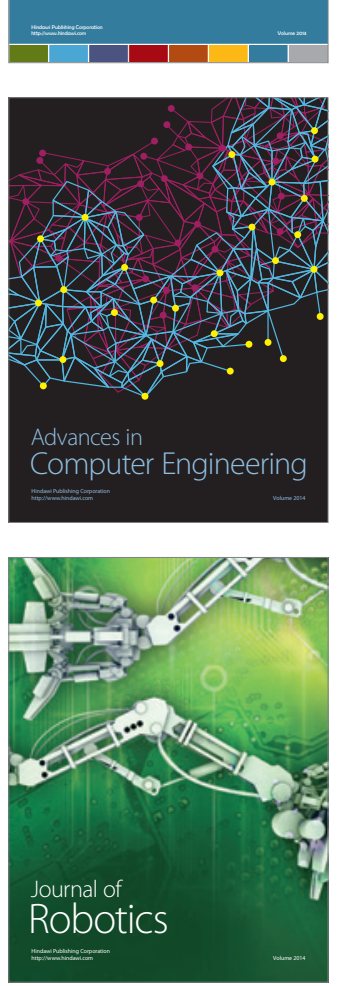
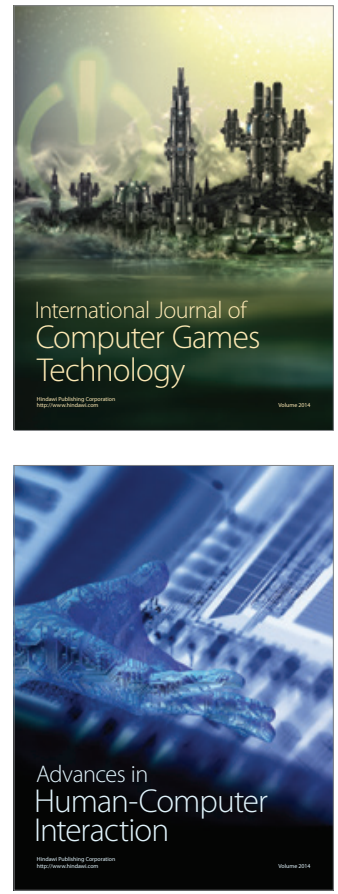
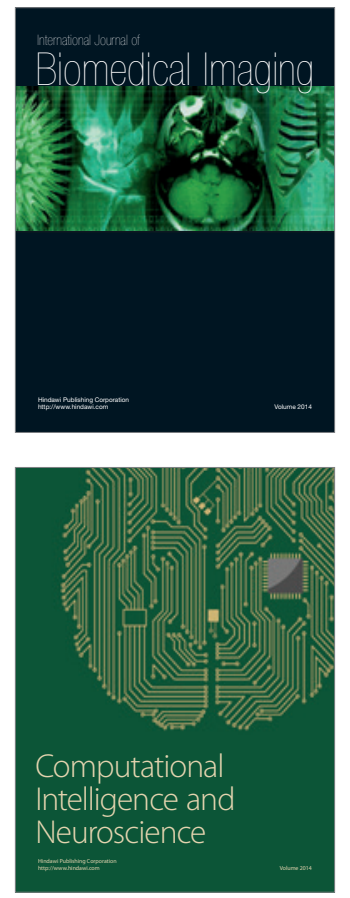
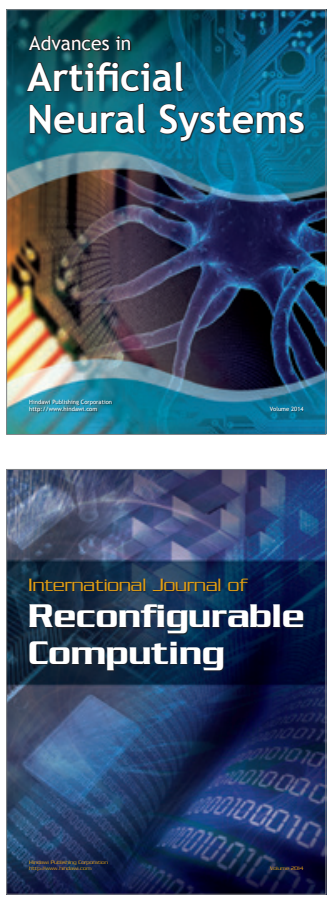
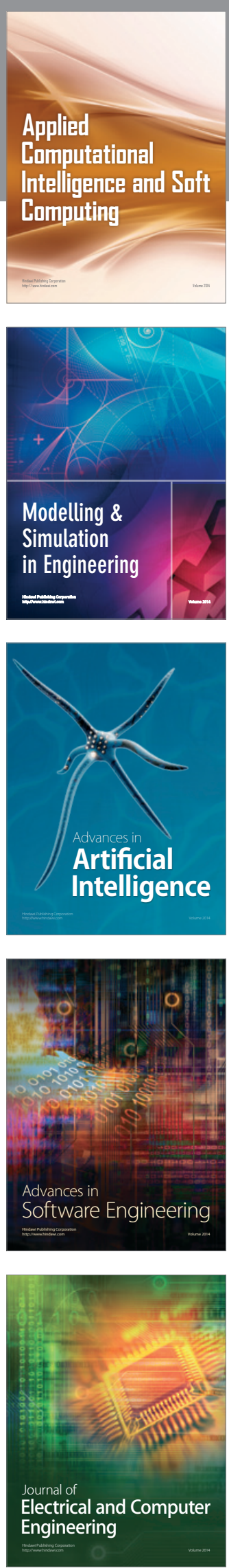